\title{
Dual Activation of CD95/CXCR4 in HEK293T cells by Abscisic Acid- and Gibberellin-inducible dCas9s (Transient Transfection)
}

Lei S Qi ( $\nabla$ stanley.qi@stanford.edu )

Stanford University

Yuchen Gao

Stanley Qi Lab, Stanford University

\section{Method Article}

Keywords: CRISPR, Cas9, Gene regulation, Synthetic biology, Inducible

Posted Date: October 25th, 2016

DOI: https://doi.org/10.1038/protex.2016.073

License: (c) (i) This work is licensed under a Creative Commons Attribution 4.0 International License.

Read Full License 


\section{Abstract}

The use of dCas9-based transcriptional activators and repressors facilitates manipulation of endogenous gene expression. However, precise temporal control of their activities and independent control of discrete gene targets in the same cell remain difficult. Here, we describe a method for utilizing orthogonal and small-molecule inducible dCas9-based transcriptional regulators for independently activating expression of CD95 and CXCR4 in HEK293T cells via transient transfection. This protocol can be easily adapted for different gene targets by using alternative sgRNAs, providing a customizable platform for flexible gene regulation.

\section{Introduction}

The bacterial CRISPR/Cas9 platform has seen rapid adoption for genome engineering in cell culture and _in vivo_ systems ${ }^{1-4}$. Previous work has shown that a mutant nuclease-dead Cas9 $\backslash(\mathrm{dCas} 9)$ can be fused to transcriptional activator or repressor domains to activate or repress genes in mammalian cells ${ }^{5-9}$. As described in the associated publication, we have recently engineered orthologous dCas 9 activators derived from two bacterial species,_S. pyogenes_ and_S. aureus_, each regulated at the protein level by a small molecule inducer. These inducers, abscisic acid ${ }^{10}$ and gibberellin ${ }^{11}$, originate from plant hormone signaling pathways and, in our system, triggers dimerization of dCas 9 with the transcriptional effector domain. We showed that these inducible dCas 9 regulators can act without crosstalk in the same cell to independently regulate expression of discrete gene targets. Here, we provide the protocol for the simultaneous use of two dCas9-based transcriptional regulators in the same cell for precise temporal manipulation of gene expression. In brief, plasmids containing the dCas9s described above along with those containing sgRNAs targeting the genes of interest are co-transfected into the target cell line. The cells are induced with the commercially-available small molecule inducers to trigger gene manipulation. The sgRNAs in our protocol target the genes_CXCR4_ and_CD95_ I(_FAS_), but can be readily adapted for alternative gene targets by changing the $\sim 20$ base pair spacer sequence for your gene of interest.

\section{Reagents}

**Plasmids \(Request from "Addgene: Stanley Qi Lab Plasmids":http://www.addgene.org/Stanley_Qi/)** 1. pPB: pCAG-GID1-VPR-IRES-Puro-WPRE pPGK-GAI-tagBFP-Sp dCas9 2. pPB: pCAG-PYL1-VPR-IRES-PuroWPRE-SV4OPA pPGK-ABI-tagBFP-Sa dCas9 3. pHR: pU6-Sp sgCD95-1 pCMV-EGFP 4. pHR: pU6-Sp sgCD95-2 pCMV-EGFP 5. pHR: pU6-Sp sgCD95-3 pCMV-EGFP 6. pHR: pU6-Sa sgv2CXCR4-1 pCMV-EGFP 7. pHR: pU6-Sa sgv2CXCR4-2 pCMV-EGFP 8. pHR: pU6-Sa sgv2CXCR4-3 pCMV-EGFP ${ }^{\star \star C}$ Cell line ${ }^{\star \star} 9$.

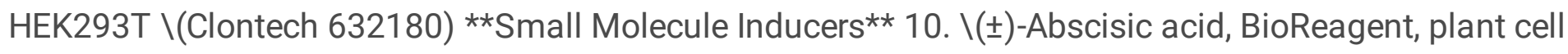
culture tested, $=98.5 \% \backslash(\mathrm{HPLC}) \backslash($ Sigma-Aldrich A1049-100MG) 11. Gibberellic Acid Acetoxymethyl Ester $\backslash$ (Santa Cruz Biotechnology sc-490123) **Antibodies** 12. PE anti-human CD95 (Fas) \[DX2] 25 tests \ (BioLegend 305607) 13. PE Mouse IgG1, $\mathrm{K}$ Isotype Ctrl \[MOPC-21] $25 \mu \mathrm{g} \backslash($ BioLegend 400111) 14. APC anti-human CD184 \(CXCR4) \[12G5] 100 tests \(BioLegend 306510) 15. APC Mouse IgG2b, $\mathrm{k}$ Isotype Ctrl $\backslash[$ MPC-11] 100 tests $\backslash($ BioLegend 400322) **Additional Reagents** 16. Mirus Bio TransIT-LT1 
Transfection Reagent \(Mirus MIR2306) 17. Opti-MEM® I Reduced Serum Medium \(Life Technologies 31985-062) 18. Tet System Approved FBS \(Clontech 631106) 19. DMEM, high glucose, GlutaMAX ${ }^{\mathrm{TM}}$ Supplement, pyruvate \(Life Technologies 10569044) 20. Versene Solution \(Life Technologies 15040066) 21. Dimethyl sulfoxide, $>=99.7 \%$, Hybri-Max $(T M)$, sterile-filtered, hybridoma tested $\backslash($ Sigma-Aldrich D2650-100ML) 22. DPBS, no calcium, no magnesium \(Life Technologies 14190-250)

\section{Equipment}

1. Tissue Culture Hood/Biosafety Cabinet $\mathrm{CO} 2$ incubator at $37^{\circ} \mathrm{C}$ and $5 \% \mathrm{CO} 2$ for mammalian cell culture 2. Microcentrifuge 3. Hemocytometer 4. Manual Pipettes \(P2, P20, P200, P1000) 5. Pipette Tips 6. 12 well CORNING $\backslash(R)$ COSTAR $\backslash(R)$ CELL CULTURE PLATE \(Fisher Scientific 07-200-82) 7. Fisherbrand Premium Microcentrifuge Tubes; Natural; 1.5mL; O.D. x L: 10.8 x 40.6mm; 500/PK \(Fisher Scientific NC0479354)

\section{Procedure}

**Transfection of Target Cells with dCas 9 and sgRNA Expression Vectors** 1. One day prior to transfection, seed 12-well cell culture plates with $6 \times 10^{4}$ HEK293T cells in $1 \mathrm{~mL}$ DMEM/GlutaMAX supplemented with $10 \%$ FBS per well. Incubate at $37^{\circ} \mathrm{C}$ and $5 \%$ CO2. 2. Twenty-four hours after plating cells, prepare the transfection complexes, scaling up the mixture masses and volumes as needed: i. For each well, combine the following expression vector DNAs. 250 ng pPB: pCAG-GID1-VPR-IRES-Puro-WPRE pPGK-GAI-tagBFP-Sp dCas9 250 ng pPB: pCAG-PYL1-VPR-IRES-Puro-WPRE-SV4OPA pPGK-ABI-tagBFP-Sa dCas9 83.3 ng pHR: pU6-Sp sgCD95-1 pCMV-EGFP 83.3 ng pHR: pU6-Sp sgCD95-2 pCMV-EGFP 83.3 ng pHR: pU6-Sp sgCD95-3 pCMV-EGFP 83.3 ng pHR: pU6-Sa sgv2CXCR4-1 pCMV-EGFP 83.3 ng pHR: pU6-Sa sgv2CXCR4-2 pCMV-EGFP 83.3 ng pHR: pU6-Sa sgv2CXCR4-3 pCMV-EGFP ii. Add this $1 \mu \mathrm{g}$ DNA mixture to $100 \mu \mathrm{L}$ Opti-MEM Reduced-Serum Medium in a microcentrifuge tube. Mix well by pipetting up and down. iii. Add $3 \mu \mathrm{L}$ Mirus TransIT-LT1 Transfection Reagent into the same tube. Mix well by pipetting up and down. iv. Allow transfection complexes to form for $30 \mathrm{~min}$ at room temperature. 3. Add $100 \mu \mathrm{L}$ of the transfection mixture to each well of the 12-well plate containing plated HEK293T cells. Mix well by rocking the plate back and forth. Incubate $24 \mathrm{~h}$ at $37^{\circ} \mathrm{C}$ and $5 \% \mathrm{CO} 2$. ${ }^{*}$ Induction of Target Cells with Abscisic acid/Gibberellin** 4. Twenty-four hours after transfection, prepare small molecules for induction as follows: Prepare 500x concentrated stocks of abscisic acid and gibberellin. Abscisic acid: Dissolve $13.22 \mathrm{mg}$ abscisic acid in $1 \mathrm{~mL}$ DMSO to make a $50 \mathrm{mM}$ solution. Gibberellin: Dissolve $2.09 \mathrm{mg}$ gibberellic acid acetyoxymethyl ester in $1 \mathrm{~mL}$ DMSO to make a $5 \mathrm{mM}$ solution. These stocks can be stored at $-20^{\circ} \mathrm{C}$. 5 . Add $2 \mu \mathrm{L}$ of the $500 \mathrm{x}$ concentrated stocks of abscisic acid and/or gibberellin to each well of the 12-well plate containing transfected HEK293T cells. 6 . Add DMSO in parallel such that $4 \mu \mathrm{L}$ total volume of $\backslash($ DMSO + abscisic acid + gibberellin) are added to each well. Mix well by rocking the plate back and forth. Incubate $48 \mathrm{~h}$ at $37^{\circ} \mathrm{C}$ and $5 \% \mathrm{CO} 2$. ${ }^{*}$ Immunostaining for CD95/CXCR4 ${ }^{\star *} 7$. Fortyeight hours after induction, harvest cells as follows: i. Aspirate cell culture media from each well. ii. Add $300 \mu \mathrm{L}$ Versene solution per well, and incubate $5 \mathrm{~min}$ at $37^{\circ} \mathrm{C}$ and $5 \% \mathrm{CO} 2$ to dissociate cells. 8. Transfer 
Versene solution containing dissociated cells into one microfuge tube per well. Centrifuge 2 min at 3,500 RPM in a microcentrifuge. 9. Prepare a blocking buffer containing 10\% FBS in DPBS. 10. Aspirate Versene solution from pelleted cells, taking care not to disturb the cell pellet. Resuspend cells in $375 \mu \mathrm{L}$ blocking buffer by pipetting up and down. Centrifuge $2 \mathrm{~min}$ at 3,500 RPM. 11. Prepare staining solutions as follows, scaling up volumes as needed: For each sample: Add $0.2 \mu \mathrm{L}$ APC-CXCR4 antibody $\backslash(1: 500$, final concentration $0.4 \mu \mathrm{g} / \mathrm{mL})$ and $0.5 \mu \mathrm{L}$ PE-CD95 antibody $\backslash(1: 200$, final concentration $0.5 \mu \mathrm{g} / \mathrm{mL})$ to $100 \mu \mathrm{L}$ $10 \%$ FBS in PBS. For isotype controls: Add $0.2 \mu \mathrm{L}$ APC IgG2 antibody $\backslash(1: 500$, final concentration 0.4 $\mu \mathrm{g} / \mathrm{mL}$ ) or $0.25 \mu \mathrm{L} P E \lg 1$ antibody $\backslash(1: 400$, final concentration $0.5 \mu \mathrm{g} / \mathrm{mL})$ to $100 \mu \mathrm{L} 10 \% \mathrm{FBS}$ in PBS. 12. Aspirate blocking buffer from pelleted cells, taking care not to disturb the cell pellet. Resuspend cells in $100 \mu \mathrm{L}$ staining solution by pipetting up and down. 13. Incubate cells $1 \mathrm{~h}$ at room temperature protected from light. Centrifuge $2 \mathrm{~min}$ at 3,500 RPM. 14. Aspirate staining solution from pelleted cells, taking care not to disturb the cell pellet. Resuspend cells in $375 \mu \mathrm{L}$ blocking buffer by pipetting up and down. Centrifuge 2 min at 3,500 RPM. 15. Resuspend cells in $150 \mu \mathrm{L}$ blocking buffer and immediately analyze by flow cytometry.

\section{Timing}

The complete protocol requires 5 working days to complete. The approximate time requirements for different days/sections of the process are as follows: Cell seeding: 20 min Transfection: 1 hour Induction of target cells: 15 min Immunostaining: 3 hours

\section{Troubleshooting}

See Table 1 for troubleshooting advice.

\section{Anticipated Results}

Figure 1 shows representative contour plots of CXCR4 and CD95 expression quantified by flow cytometry in HEK293T cells transfected with the indicated constructs and induced with ABA and/or GA. The cells were gated for tagBFP and EGFP prior to analysis to identify positive expression of dCas9 and sgRNA constructs.

\section{References}

1. Jinek, M. et al. A programmable dual-RNA-guided DNA endonuclease in adaptive bacterial immunity. Science 337, 816-21 \(2012). 2. Mali, P. et al. RNA-guided human genome engineering via Cas9. Science 339, 823-6 \(2013). 3. Cong, L. et al. Multiplex genome engineering using CRISPR/Cas systems. Science 339, 819-23 \(2013). 4. Jiang, W., Bikard, D., Cox, D., Zhang, F. \& Marraffini, L. A. RNA-guided editing of bacterial genomes using CRISPR-Cas systems. Nat. Biotechnol. 31, 233-9 \(2013). 5. Gilbert, L. A. et al. CRISPR-mediated modular RNA-guided regulation of transcription in eukaryotes. Cell 154, 442-51 I (2013). 6. Maeder, M. L. et al. CRISPR RNA-guided activation of endogenous human genes. Nat. Methods 
10, 977-9 \(2013). 7. Perez-Pinera, P. et al. RNA-guided gene activation by CRISPR-Cas9-based transcription factors. Nat. Methods 10, 973-6 \(2013). 8. Mali, P. et al. CAS9 transcriptional activators for target specificity screening and paired nickases for cooperative genome engineering. Nat. Biotechnol. 31, 833-8 \(2013). 9. Cheng, A. W. et al. Multiplexed activation of endogenous genes by CRISPR-on, an RNAguided transcriptional activator system. Cell Res. 23, 1163-71 \(2013). 10. Liang, F.-S., Ho, W. Q. \& Crabtree, G. R. Engineering the ABA plant stress pathway for regulation of induced proximity. Sci. Signal. 4, rs2 \(2011). 11. Miyamoto, T. et al. Rapid and orthogonal logic gating with a gibberellin-induced dimerization system. Nat. Chem. Biol. 8, 465-70 \(2012).

\section{Figures}




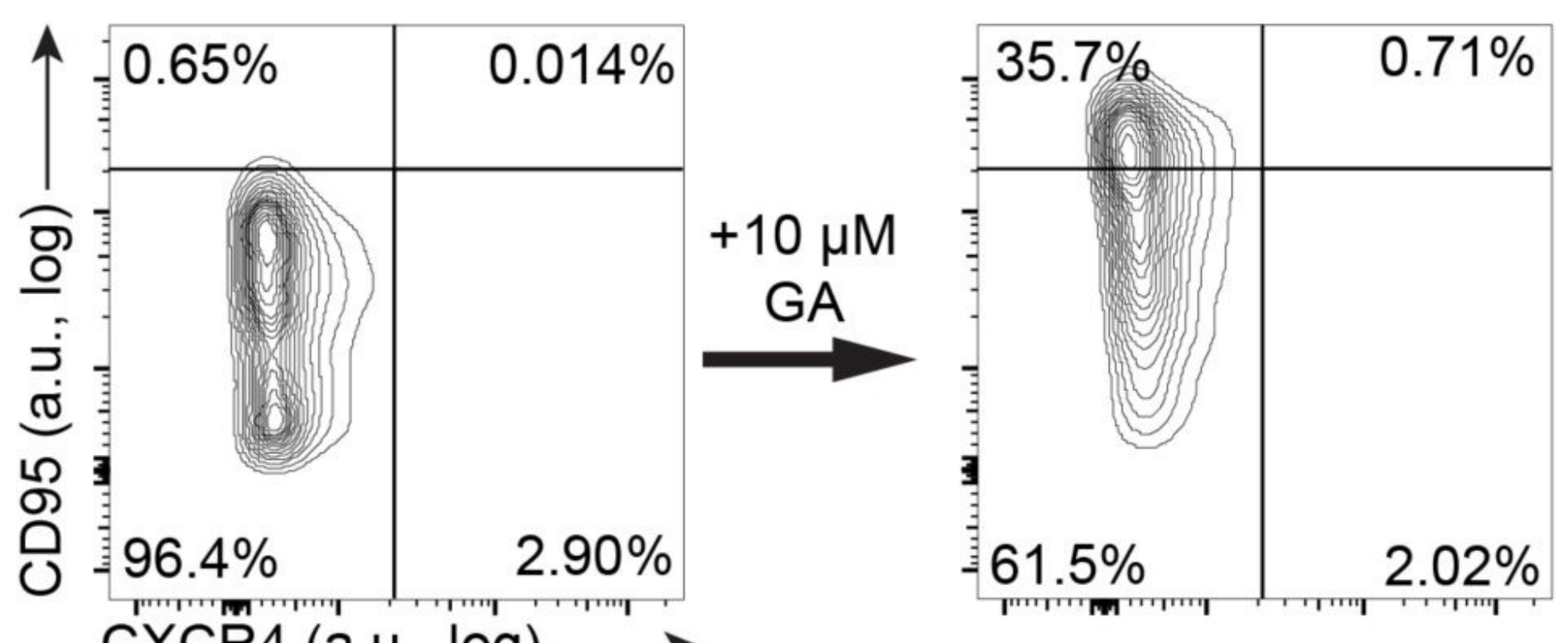

CXCR4 (a.u., log) $\longrightarrow$

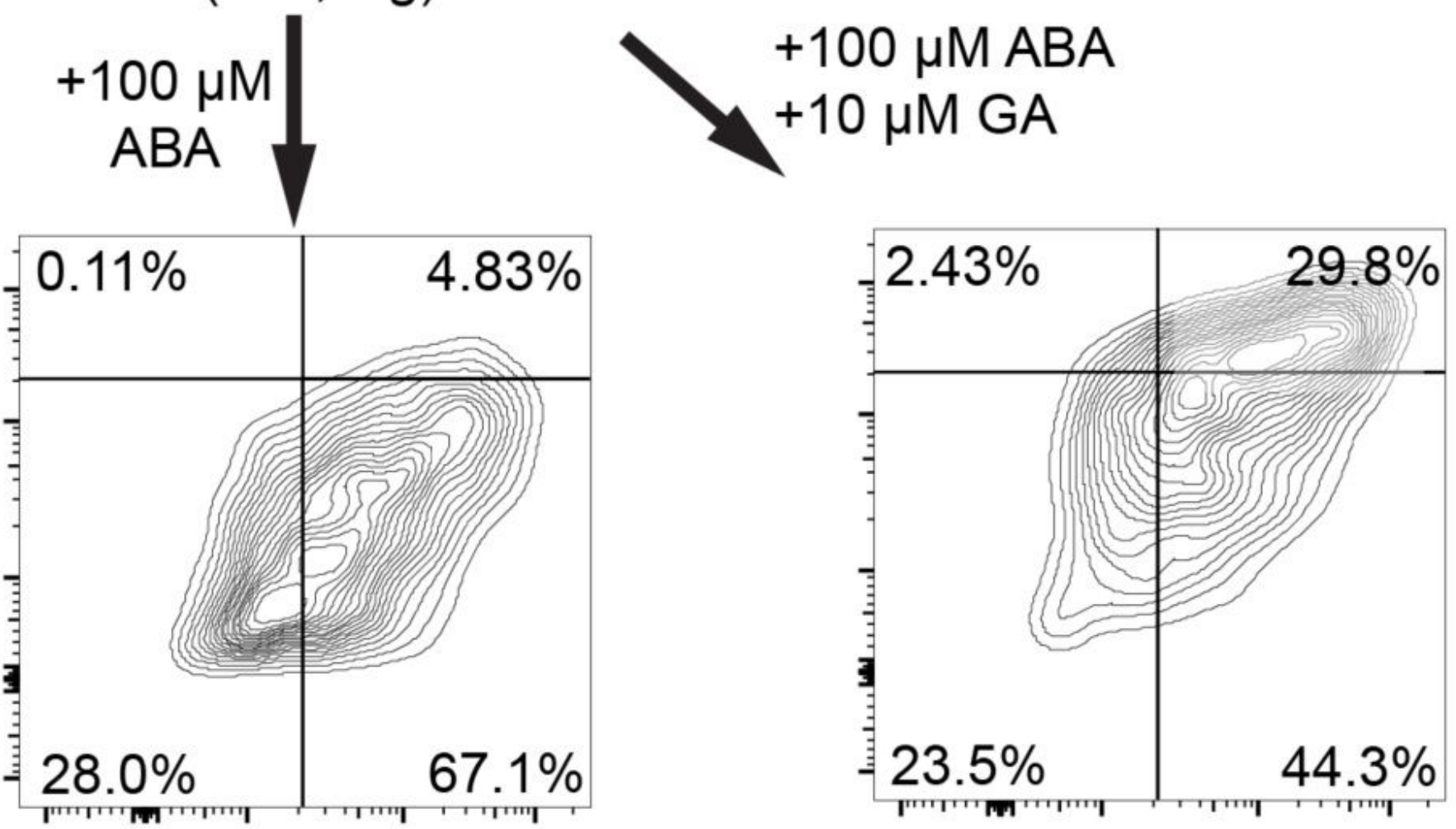

Figure 1

CD95/CXCR4 Representative Quantification

\begin{tabular}{|c|c|c|c|}
\hline Problem & Possible Reason & Solution & Step(s) \\
\hline High amount of cell death observed & Cells sensitive to transfection reagent & Reduce amount of Mirus TransIT-LT1 Transfection Reagent used & 2 \\
\hline Cells become detached during transfection & Pipetting of transfection mixture is disturbing adherent cells & Add transfection mix in a dropwise fashion to the side of each well & 3 \\
\hline No staining observed & Incubation time or concentration of antibody too low & Increase incubation time and/or antibody concentration & 11,13 \\
\hline Excess isotype signal & Strong non-specific isotype binding & Add additional blocking buffer wash steps after antibody incubation & 14 \\
\hline
\end{tabular}

Figure 2 
Table 1 Troubleshooting 